\title{
Potential Effects of Digital Inequality on Treatment Seeking for Opioid Use Disorder
}

\author{
Renee Garett ${ }^{1} \cdot$ Sean D. Young ${ }^{2,3}$
}

Accepted: 19 August 2021 / Published online: 27 August 2021

(C) The Author(s), under exclusive licence to Springer Science+Business Media, LLC, part of Springer Nature 2021

\begin{abstract}
Despite the availability of effective treatment, medications for opioid use disorder are underutilized due to a variety of practical, political, and psychological reasons. Digital inequalities, such as limited access to technology, skills to leverage the technology for desirable outcomes, and social resources, may be contributing to negative health outcomes. In addition, broader health literacy plays an integral part in the capacity of individuals to appraise opioid medication-related online information. This paper explores the role of digital inequalities in the uptake of treatment for opioid use disorder. Given the shift toward telemedicine and online counseling for substance use treatment as a consequence of the COVID-19 pandemic, more research into the digital inequalities faced by those who misuse opioids may provide insight into ways of engaging and encouraging this population to utilize treatment.
\end{abstract}

Keywords Digital inequality · Health literacy · Medications for opioid use disorder · Opioid use disorder

Opioid misuse and abuse continue to plague the USA, affecting thousands of lives. Approximately 21-29\% of US patients who have been prescribed opioids for pain management misuse them, and between 8 and 12\% develop opioid use disorder (OUD) (National Institute on Drug Abuse, 2021). Deaths attributable to opioid overdose between 1999 and 2019 approximated 500,000 (Centers for Disease Control \& Prevention, 2021), with fatalities from synthetic opioid increasing 55\% between 2019 and 2020 (Mann, 2021). Globally, approximately 58 million people used opioids in 2018, and to date, opioids contributed to more than $70 \%$ of the 0.5 million deaths related to drug use (WHO. World Health Organization, 2020). Effective medication treatments exist to treat OUD (MOUD); however, uptake for treatment is low due to factors including logistical, attitudinal, geographical, regulatory, and financial (National Academies of Sciences et al., 2019). In addition, stigma

Sean D. Young

syoung5@hs.uci.edu

1 ElevateU, Irvine, CA, USA

2 Department of Emergency Medicine, University of California Irvine, CA, Irvine, USA

3 University of California Institute for Prediction Technology, Department of Informatics, University of California Irvine, CA, Irvine, USA 
from both providers and patients plays a significant obstacle in uptake of treatment (Tsai et al., 2019).

To increase patient initiation of MOUDs, patients will need to have better access to information about them. Social media and online forums such as Reddit (D'Agostino et al., 2017) and Facebook (Young \& Heinzerling, 2017; Young et al., 2018a, 2020) have been outlets for patients with OUD to share experiences with others. Given the stigma that individuals with OUD experience and their interest in using the Internet to search for OUD treatments (Young et al., 2018b, 2021), these types of online outlets might be tools for patients with OUD to use. However, digital inequality may inadvertently result in hindering uptake to treatment. In this paper, we seek to explore the effects of digital inequality on seeking treatment among those with OUD.

\section{Digital Inequality and Health Literacy}

Digital inequality refers to differences and limitations in people's access to and use of digital technologies in their daily life. Kang and Pamukcu (2009) stated that digital inequality manifests itself through disparities in five areas: technical means (i.e., access to bandwidth), autonomy (i.e., using technology independently), skill (i.e., seek/download information), social support (i.e., connecting with experienced users), and purpose (i.e., leveraging technology for improved outcomes) (Kang \& Pamukcu, 2009).

With respect to access, a recent study by Pew Research Center found that broadband access was lower for Blacks and Hispanics, low income, and those living in rural areas (Pew Research Center, 2021), though smartphone ownership has assisted with Internet access for Blacks and Hispanics (Perrin \& Turner, 2019) to minimize the digital divide. Additionally, studies have found that individuals who lack one type of digital skill (e.g., downloading files or bookmarking a website) are likely to lack others (e.g., being able to use the correct keywords in Internet searches) (Deursen et al., 2017). Although limited research has studied whether and how racial/ethnic groups have less access to information about opioids and MOUDs, these data on broader lack of access to health information suggests that racial/ethnic groups with OUD might similarly have less access to information about MOUD as a result of digital inequality.

Beyond access to information, digital health literacy is important to ensure that patients with access are able to understand online health-related information. Health literacy is the ability to comprehend and process health-related information for improved health outcomes (WHO. World Health Organization, n.d.-b), with lower health literacy associated with decreased preventive behaviors and poor health outcomes such as more hospitalizations, poorer overall health status and higher mortality among elderly patients, lower preventive behaviors such as screening and vaccination, and poorer understanding of health information (McDonald \& Shenkman, 2018). Among chronic care patients, lower health literacy was associated with higher opioid misuse above other covariates such as mental health, age, gender, education, and income (Rogers et al., 2020). Digital health or ehealth literacy adds a technological component to the health literacy construct, which includes a person's ability to seek and find, in addition to comprehend and appraise, information using electronic sources and the application of that knowledge to address health issues (Norman \& Skinner, 2006). Studies examining the effects of digital health literacy have found that 
higher digital health literacy translated to better knowledge and protective health behaviors (An et al., 2021) and online health-seeking behaviors (Wong \& Cheung, 2019).

\section{Potential Effects of Digital Inequity on Treatment Uptake for MOUD}

As a result of digital inequity, people with OUD might not have access to information about treatments. Among those already enrolled in MOUD treatment programs, recent studies found that smartphone and computer ownership, as well as Internet use, were high, even among nonurban residents, suggesting that access to digital technologies might play a role (Ashford et al., 2018; Masson et al., 2019; Winstanley et al., 2018). Internet use for health-related activities was related to younger age, current employment, less bodily pain, and higher acceptance for Internet use of mobile technology (Masson et al., 2019). High access to technology and Internet use may be beneficial as healthcare provision shifted to online therapy and telemedicine during the COVID-19 pandemic (Telemedicine for Substance Use Disorders, n.d.). This also has implications for participation in mutual help groups such as Narcotics Anonymous that offers phone and online meetings (Virtual NA, n.d.). However, more studies are needed to determine digital inequalities among individuals who misuse opioids or who have OUD and are not in treatment programs. Given the lower rate of broadband access for some segments of the population (rural), an understanding of access to the Internet may offer insight to treatment options for specific key populations, especially as they pertain to options when treatment facilities are scarce.

Patients with OUD also need to have sufficient digital health literacy to effectively understand online information about MOUDs and arrive at an informed decision about which treatment might work best for them. Although investigators have found that a high proportion of patients in treatment programs have access to the Internet through a smartphone or computer and use the Internet for health-related activities (Masson et al., 2019), few studies have explored digital health literacy and the capacity of participants to comprehend health-related materials they viewed. More studies are needed to explore whether and how patients are able to understand online information about opioids and related treatments, including identifying reputable sites versus sites with misinformation. A recent review of opioid-related mobile applications (apps) found that although the apps were in line with strategies to address the opioid crisis through prevention, treatment, and overdose, few met quality standards (Vilardaga et al., 2020). As the numbers of opioid-related apps increase, digital literacy, including the ability to understand the purpose of the app and how to use it properly, will contribute to how well users will benefit from the app.

Low health literacy may reduce people's ability to distinguish between information that is factual from misinformation, both from online and offline sources. A study found that health literacy was low among patients in an opioid treatment program, and although participants were confident in understanding instructions from physicians and pharmacists and health warnings about negative health behaviors, a lower proportion stated that judging information from the media about health risk or how to become healthy was very easy (Dahlman et al., 2020). A review by Kim and Xie on online health services use found that in studies that examined the relationship between health literacy and online seeking behavior, even those participants with high health literacy had low self-efficacy in their ability to find health-related information online or were 
uncertain about the quality of the information they found (Kim \& Xie, 2017). Among persons with HIV, low digital health literacy translated to low confidence in seeking information online and preferred to obtain information from their healthcare provider (Nokes et al., 2018).

\section{Future Research}

As stated above, there is very limited research that has been done to examine the effects of digital inequalities and digital literacy on treatment decisions for OUD. Public health departments and opioid researchers may benefit from gaining this information to better understand and address some of the barriers to low uptake of MOUDs. The knowledge, attitudes, and behaviors (both online and offline) of those who are currently in treatment programs may be different from those who are not, which warrants examination. Researchers may also collaborate with clinicians and other healthcare providers in this effort to help providers identify patients who would most benefit from (digital) health literacy education and training, as well as educational institutions and community organizations. Although efforts and strategies have been proposed to improve health through digital innovation and access (WHO. World Health Organization, n.d.-a), much work needs to be done in learning from those who will benefit from these technologies. Continued efforts by policy makers to regulate information presented online, especially as it pertains to health and treatment, and provide funding to meet programmatic needs may assist in this endeavor.

\section{Conclusion}

Digital inequalities and digital health literacy might be contributing to the low rates of MOUD initiation among patients with OUD. With patients' increasing interest in seeking health information online, including patients with OUD, Internet-based technologies will play an increasing role in OUD prevention and treatment. However, few studies have examined the role of access to and understanding of online information about MOUDs, creating a need for additional research on this topic.

Author Contribution All authors contributed to the manuscript conception and design. All authors read and approved the final manuscript.

Funding This study was funded by the National Center for Complementary and Integrative Health [4R33AT010606-03], National Institute on Drug Abuse (NIDA), and National Institute of Allergy and Infectious Diseases (NIAID).

\section{Declarations}

Conflict of Interest The authors declare no competing interests. 


\section{References}

An, L., Bacon, E., Hawley, S., Yang, P., Russell, D., Huffman, S., \& Resnicow, K. (2021). Relationship between coronavirus-related eHealth literacy and COVID-19 knowledge, attitudes, and practices among US adults: Web-based survey study. Journal of Medical Internet Research, 23(3), e25042. https://doi.org/10.2196/25042

Ashford, R. D., Lynch, K., \& Curtis, B. (2018). Technology and social media use among patients enrolled in outpatient addiction treatment programs: Cross-sectional survey study. Journal of Medical Internet Research, 20(3), e9172. https://doi.org/10.2196/jmir.9172

Centers for Disease Control and Prevention. (2021, March 17). Understanding the epidemic. CDC.Gov. https://www.cdc.gov/drugoverdose/epidemic/index.html

D’Agostino, A. R., Optican, A. R., Sowles, S. J., Krauss, M. J., Escobar Lee, K., \& Cavazos-Rehg, P. A. (2017). Social networking online to recover from opioid use disorder: A study of community interactions. Drug and Alcohol Dependence, 181, 5-10. https://doi.org/10.1016/j.drugalcdep.2017.09.010

Dahlman, D., Ekefäll, M., \& Garpenhag, L. (2020). Health literacy among Swedish patients in opioid substitution treatment: A mixed-methods study. Drug and Alcohol Dependence, 214, 108186. https://doi. org/10.1016/j.drugalcdep.2020.108186

Deursen, A. J. A. M., Helsper, E., Eynon, R., \& Van Dijk, J. A. G. M. (2017). The compoundness and sequentiality of digital inequality. International Journal of Communication, 2017, 452-473.

Kang, S., \& Pamukcu, A. (2009). Digital inequality: Information poverty in the information age (p. 12). The Greenlining Institute. https://ecfsapi.fcc.gov/file/7020246470.pdf

Kim, H., \& Xie, B. (2017). Health literacy in the eHealth era: A systematic review of the literature. Patient Education and Counseling, 100(6), 1073-1082. https://doi.org/10.1016/j.pec.2017.01.015

Mann, B. (2021, April 22). During pandemic, fentanyl's spread made illicit drug use far more treacherous: Shots - Health news : NPR. NPR.Org. https://www.npr.org/sections/health-shots/2021/04/22/98983 3102/overdose-deaths-surged-in-pandemic-as-more-drugs-were-laced-with-fentanyl

Masson, C. L., Chen, I. Q., Levine, J. A., Shopshire, M. S., \& Sorensen, J. L. (2019). Health-related internet use among opioid treatment patients. Addictive Behaviors Reports, 9, 100157. https://doi.org/10. 1016/j.abrep.2018.100157

McDonald, M., \& Shenkman, L. (2018). Health literacy and health outcomes of adults in the United States: Implications for providers. https://purdueglobal.dspacedirect.org/handle/20.500.12264/20

National Academies of Sciences, E., Division, H. and M., Policy, B. on H. S., Disorder, C. on M.-A. T. for O. U., Mancher, M., \& Leshner, A. I. (2019). Barriers to broader use of medications to treat opioid use disorder. In Medications for Opioid Use Disorder Save Lives. National Academies Press (US). https:// www.ncbi.nlm.nih.gov/books/NBK541389/

National Institute on Drug Abuse. (2021, February 25). Opioid overdose crisis. National Institute on Drug Abuse. https://www.drugabuse.gov/drug-topics/opioids/opioid-overdose-crisis

Nokes, K. M., Reyes, D. M., \& Hickey, D. E. (2018). Perceptions of low income HIV+ persons about finding accurate web-based health information. Applied Nursing Research, 42, 70-76. https://doi.org/10. 1016/j.apnr.2018.06.002

Norman, C. D., \& Skinner, H. A. (2006). eHealth literacy: Essential skills for consumer health in a networked world. Journal of Medical Internet Research, 8(2), e506. https://doi.org/10.2196/jmir.8.2.e9

Perrin, A., \& Turner, E. (2019, August 20). Smartphones help blacks, Hispanics bridge some - but not all - digital gaps with whites. Pew Research Center. https://www.pewresearch.org/fact-tank/2019/08/20/ smartphones-help-blacks-hispanics-bridge-some-but-not-all-digital-gaps-with-whites/

Pew Research Center. (2021, April 7). Demographics of Internet and home broadband usage in the United States. Pew Research Center. https://www.pewresearch.org/internet/fact-sheet/internet-broadband/

Rogers, A. H., Bakhshaie, J., Orr, M. F., Ditre, J. W., \& Zvolensky, M. J. (2020). Health literacy, opioid misuse, and pain experience among adults with chronic pain. Pain Medicine, 21(4), 670-676. https:// doi.org/10.1093/pm/pnz062

Telemedicine for substance use disorders: Moving essential substance use interventions online. (n.d.). Research Impact Indiana University. Retrieved October 26, 2020, from https://research.impact.iu.edu/ coronavirus/telehealth.html

Tsai, A. C., Kiang, M. V., Barnett, M. L., Beletsky, L., Keyes, K. M., McGinty, E. E., Smith, L. R., Strathdee, S. A., Wakeman, S. E., \& Venkataramani, A. S. (2019). Stigma as a fundamental hindrance to the United States opioid overdose crisis response. PLoS Medicine, 16(11). https://doi.org/10.1371/journal. pmed.1002969

Vilardaga, R., Fisher, T., Palenski, P. E., Kumaresan, V., Mannelli, P., Sweitzer, M. M., McClernon, F. J., Engelhard, M. M., Sabo, P. L., \& Garrison, K. A. (2020). Review of popularity and quality standards 
of opioid-related smartphone apps. Current Addiction Reports, 7(4), 486-496. https://doi.org/10.1007/ s40429-020-00344-6

Virtual NA. (n.d.). Retrieved April 27, 2021, from https://virtual-na.org/

WHO. World Health Organization. (n.d.-a). Global strategy on digital health 2020-2025. World Health Organization. https://www.who.int/docs/default-source/documents/gs4dhdaa2a9f352b0445bafbc79ca7 99dce4d.pdf?sfvrsn=f112ede5_38

WHO. World Health Organization. (n.d.-b). Health literacy. WHO; World Health Organization. Retrieved April 29, 2021, from http://www.who.int/healthpromotion/health-literacy/en/

WHO. World Health Organization. (2020, August 28). Opioid overdose. https://www.who.int/news-room/ fact-sheets/detail/opioid-overdose

Winstanley, E. L., Stroup-Menge, B., \& Snyder, K. (2018). The promise of technology-based services for addiction treatment clients residing in nonurban areas. Journal of Studies on Alcohol and Drugs, 79(3), 503-504. https://doi.org/10.15288/jsad.2018.79.503

Wong, D.K.-K., \& Cheung, M.-K. (2019). Online health information seeking and eHealth literacy among patients attending a primary care clinic in Hong Kong: A cross-sectional survey. Journal of Medical Internet Research, 21(3), e10831. https://doi.org/10.2196/10831

Young, S. D., \& Heinzerling, K. (2017). The harnessing online peer education (HOPE) intervention for reducing prescription drug abuse: A qualitative study. Journal of Substance Use, 22(6), 592-596. https://doi.org/10.1080/14659891.2016.1271039

Young, S. D., Koussa, M., Lee, S. J., Perez, H., Gill, N., Gelberg, L., \& Heinzerling, K. (2018a). Feasibility of a social media/online community support group intervention among chronic pain patients on opioid therapy. Journal of Addictive Diseases, 37(1-2), 96-101. https://doi.org/10.1080/10550887.2018. 1557992

Young, S. D., Zheng, K., Chu, L. F., \& Humphreys, K. (2018b). Internet searches for opioids predict future emergency department heroin admissions. Drug and Alcohol Dependence, 190, 166-169. https://doi. org/10.1016/j.drugalcdep.2018.05.009

Young, S. D., Lee, S. J., Perez, H., Gill, N., Gelberg, L., \& Heinzerling, K. (2020). Social media as an emerging tool for reducing prescription opioid misuse risk factors. Heliyon, 6(3), e03471. https://doi. org/10.1016/j.heliyon.2020.e03471

Young, S. D., Zhang, Q., Zhou, J. et al. (2021). Internet search and medicaid prescription drug data as predictors of opioid emergency department visits. NPJ Digital Medicine, 4, 21. https://doi.org/10.1038/ s41746-021-00392-w

Publisher's Note Springer Nature remains neutral with regard to jurisdictional claims in published maps and institutional affiliations. 